\title{
Locality Estimates for Quantum Spin Systems
}

\author{
Bruno Nachtergaele \\ Department of Mathematics \\ University of California at Davis \\ Davis CA 95616, USA \\ Email: bxn@math.ucdavis.edu \\ and \\ Robert Sims \\ Faculty of Mathematics \\ University of Vienna \\ 1090 Wien, Austria \\ Email: robert.sims@univie.ac.at
}

Version: September 3, 2018

\begin{abstract}
We review some recent results that express or rely on the locality properties of the dynamics of quantum spin systems. In particular, we present a slightly sharper version of the recently obtained Lieb-Robinson bound on the group velocity for such systems on a large class of metric graphs. Using this bound we provide expressions of the quasi-locality of the dynamics in various forms, present a proof of the Exponential Clustering Theorem, and discuss a multi-dimensional Lieb-Schultz-Mattis Theorem.
\end{abstract}

\section{Introduction}

Locality is a fundamental property of all current physical theories. Sets of observables can be associated with points or bounded regions in space or space-time and a relativistic dynamics will preserve this structure [8]. How the locality property manifests itself mathematically in important situations continues to be an active topic of investigation [13.

There is a wide range of important physical systems, however, which we prefer to describe by very effective non-relativistic quantum theories with Hamiltonian dynamics. Even if the Hamiltonian has only finite-range interactions, the dynamics it generates generally does not preserve locality, i.e., there is no strict equivalent to the finite speed of light. However, locality still holds in an approximate sense, and there is an associated finite velocity, which is sometimes referred to as the group velocity. We call it the Lieb-Robinson velocity since Lieb and Robinson were the first to prove its existence and to obtain a bound for it [16]. They proved that to a high degree

\footnotetext{
${ }^{*}$ Copyright (C) 2007 by the authors. This paper may be reproduced, in its entirety, for non-commercial purposes.
} 
of accuracy locality is preserved by quantum spin dynamics in the sense that any local observable evolved for a time $t>0$ remains localized in a region of space with diameter proportional to $t$, up to an arbitrarily small correction. This also means that spatial correlations between observables separated by a distance $d$ cannot be established faster than a time of order $d$.

The fundamental issue of locality may be sufficient motivation to extend the Lieb-Robinson bounds to more general situations, but there are other good reasons to try to generalize their result and to improve the estimates they obtained. As we will discuss below, locality, or the approximate locality of the dynamics, has been shown to be responsible for a considerable number of other important properties relevant for models of many-body systems. In many situations, however, the implications of locality have yet to be fully explored.

We will begin this note by presenting a short proof of the new Lieb-Robinson bounds obtained successively in [21], [12, and [19. This improved result we give below sharpens the bounds previously obtained in that the prefactor now only grows as the smallest surface area of the supports of the local observables. We do this in Section 2, An application where this surface area dependence, rather than volume dependence, is important can be found in [6].

In Section 3, we present two perspectives on how Lieb-Robinson bounds may be used to provide explicit estimates on the local structure of the time evolution. As a consequence, one easily derives bounds on, for example, multiple commutators and the rate at which spatial correlations can be established in normalized product states.

Section 4 discusses the so-called Exponential Clustering Theorem. In the relativistic context it has been known for a long time that a gap in the spectrum above the vacuum state implies exponential decay of spatial correlations in that state [2, 24, 7]. That a similar result should hold in the non-relativistic setting such as quantum spin systems was long expected and taken for granted by theoretical physicists [26]. In [10], Hastings proposed to use Lieb-Robinson bounds to obtain such a result and a complete proof was recently given in [21, 12].

As a final application of these locality bounds, we describe a new proof of the Lieb-SchultzMattis theorem, see [10, 22, in Section [5. These results can be traced back to [10] where Hastings introduced a new way to construct and analyze variational states for low-lying excitations of gapped Hamiltonians. He developed a notion of a quasi-adiabatic evolution [11] which he then used to present a multi-dimensional analogue of the celebrated Lieb-Schultz-Mattis theorem 15. Such a theorem is applicable, for example, to the standard spin- $1 / 2$, anti-ferromagnetic Heisenberg model and yields an upper bound on the first excited state of order $c(\log L) / L$ for systems of size $L$. His arguments rely on Lieb-Robinson bounds and the Exponential Clustering Theorem in an essential way, and we have recently obtained a rigorous proof of this result which holds in a rather general setting, see [22].

We expect that the ideas currently emerging from recent applications of Lieb-Robinson bounds will continue to lead to interesting new results for quantum spin systems in the near future.

\section{Lieb-Robinson Bounds}

The first proof of locality bounds in the context of quantum spin systems appeared in 1972 in a paper by Lieb and Robinson [16]. They proved a bound on the group velocity corresponding to the dynamics generated by a variety of short range Hamiltonians. In a series of works [21, [12, and [19], these estimates have been generalized, and the proof we provide below, see Theorem 2.1 . illustrates many of the new insights which have recently been developed.

The result stated in Theorem 2.1 below differs from that which may be found in [16] in two important ways. First, the new proof does not require the use of the Fourier transform, and 
therefore, it extends to models defined on sets without an underlying lattice structure. These results may be of interest to those who wish to study quantum spin systems in the context of quasi-crystals or in the study of circuits for quantum computation. Second, and most importantly, the constants which appear in our bound do not depend on the dimensions of the underlying, single-site Hilbert spaces. This opens up the possibility of applying them to models with an infinite-dimensional Hilbert space, such as lattice oscillators [20].

The basic set up in this theory concerns quantum spins systems, in particular, a finite or infinite number of spins labeled by $x \in V$. A finite dimensional Hilbert space $\mathcal{H}_{x}$ is assigned to each site $x \in V$. These may represent the spin of an electron, photon, or an atom. In other contexts, these states may represent the ground state and first exited state of an atom or a molecule. More abstractly, these systems may, for example, model a collection qubits, the basic units of quantum information theory and quantum computation.

If the set $V$ is finite, the Hilbert space of states is given by $\mathcal{H}_{V}=\bigotimes_{x \in V} \mathcal{H}_{x}$. For each spin $x$, the observables are the complex $n_{x} \times n_{x}$ matrices, $M_{n_{x}}$, where $n_{x}=\operatorname{dim}\left(\mathcal{H}_{x}\right)$. In this context, the algebra of observables for the whole system is $\mathcal{A}_{V}=\bigotimes_{x \in V} M_{n_{x}}$.

The locality results we wish to describe pertain to observables with finite support. Here, the support of an observable is understood as follows. If $X \subset V$, we write $\mathcal{A}_{X}=\bigotimes_{x \in X} M_{n_{x}}$. By identifying $A \in \mathcal{A}_{X}$ with $A \otimes \mathbb{1} \in \mathcal{A}_{V}$, we have that $\mathcal{A}_{X} \subset \mathcal{A}_{V}$. The support of an observable $A \in \mathcal{A}_{V}$ in the minimal set $X \subset V$ for which $A=A^{\prime} \otimes \mathbb{1}$ with $A^{\prime} \in \mathcal{A}_{X}$.

For infinite $V$, the algebra of observables is the completion of the algebra of local observables given by

$$
\mathcal{A}_{V}=\bigcup_{X \subset V} \mathcal{A}_{X}
$$

where the union is over all finite $X \subset V$.

To describe the models we wish to investigate, we must first define interactions, local Hamiltonians, and the corresponding dynamics. An interaction is a map $\Phi$ from the set of subsets of $V$ to $\mathcal{A}_{V}$ with the property that $\Phi(X) \in \mathcal{A}_{X}$ and $\Phi(X)=\Phi(X)^{*}$ for all finite $X \subset V$. A quantum spin model is defined by a family of local Hamiltonians, parametrized by finite subsets $\Lambda \subset V$, given by

$$
H_{\Lambda}^{\Phi}=\sum_{X \subset \Lambda} \Phi(X)
$$

For notational convenience, we will often drop the dependence of $H_{\Lambda}^{\Phi}$ on $\Phi$. The dynamics, or time evolution, generated by a quantum spin model is the one-parameter group of automorphisms, $\left\{\tau_{t}^{\Lambda}\right\}_{t \in \mathbb{R}}$, defined by

$$
\tau_{t}^{\Lambda}(A)=e^{i t H_{\Lambda}} A e^{-i t H_{\Lambda}}, \quad A \in \mathcal{A}_{\Lambda},
$$

which is always well defined for finite sets $\Lambda$. In the context of infinite systems, a boundedness condition on the interaction is required in order for the finite-volume dynamics to converge to a strongly continuous one-parameter group of automorphisms on $\mathcal{A}_{V}$.

The locality results we prove in Theorem 2.1 are valid for a large class of interactions. To describe this class precisely, we first put a condition on the set $V$, which is relevant only in the event that $V$ is infinite. We assume that the set $V$ is equipped with a metric $d$ and that there exists a non-increasing function $F:[0, \infty) \rightarrow(0, \infty)$ for which:

i) $F$ is uniformly integrable over $V$, i.e.,

$$
\|F\|=\sup _{x \in V} \sum_{y \in V} F(d(x, y))<\infty,
$$

and 
ii) $F$ satisfies

$$
C=\sup _{x, y \in V} \sum_{z \in V} \frac{F(d(x, z)) F(d(z, y))}{F(d(x, y))}<\infty .
$$

Given such a set $V$, it is easy to see that for any $F$ which satisfies i) and ii) above, then the family of functions $F_{a}$, for $a \geq 0$, defined by

$$
F_{a}(x)=e^{-a x} F(x)
$$

also satisfy i) and ii) with $\left\|F_{a}\right\| \leq\|F\|$ and $C_{a} \leq C$. In this context, we define the set $\mathcal{B}_{a}(V)$ to be those interactions $\Phi$ on $V$ which satisfy

$$
\|\Phi\|_{a}=\sup _{x, y \in V} \sum_{X \ni x, y} \frac{\|\Phi(X)\|}{F_{a}(d(x, y))}<\infty .
$$

The Lieb-Robinson bounds we will prove are valid for all $\Phi \in \mathcal{B}_{a}(V)$ with $a \geq 0$. Simply stated, these results correspond to estimates of the form

$$
\left\|\left[\tau_{t}^{\Lambda}(A), B\right]\right\| \leq c(A, B) e^{-\mu\left(d(A, B)-v_{\Phi}|t|\right)},
$$

where $A$ and $B$ are local observables, $\tau_{t}^{\Lambda}(\cdot)$ is the time evolution corresponding to a finite volume Hamiltonian generated by an interaction $\Phi \in \mathcal{B}_{a}(V)$, and $d(A, B)$ is the distance between the supports of $A$ and $B$. What is crucial in these estimates is that the constants $c(A, B), \mu$, and $v_{\Phi}$ are independent of the volume $\Lambda \subset V$ on which $\tau_{t}^{\Lambda}(\cdot)$ is defined. Physically, the constant $v_{\Phi}$ corresponds to a bound on the velocity at which the dynamics, generated by $\Phi$, propagates through the system.

Intuitively, it is clear that the spread of the interactions through the system should depend on the surface area of the support of the local observable $A$, typically denoted by $X$, not it's volume. To make this explicit in our bound, we will denote the surface of a set $X$, regarded as a subset of $\Lambda \subset V$, by

$$
S_{\Lambda}(X)=\left\{Z \subset \Lambda: Z \cap X \neq \emptyset \text { and } Z \cap X^{c} \neq \emptyset\right\} .
$$

Here we will use the notation $S(X)=S_{V}(X)$, and define

$$
\|\Phi\|_{a}(x ; X)=\left\{\begin{array}{cc}
\sup _{y \in \Lambda} \sum_{\substack{Z \in S(X): \\
x, y \in Z}} \frac{\|\Phi(Z)\|}{F_{a}(d(x, y))} & \text { if } x \in X \\
0 & \text { otherwise }
\end{array}\right.
$$

a quantity corresponding to the interaction terms across the surface of $X$. Comparing the local quantity appearing in (9) with the norm on the full interaction given by (6), one trivially has that

$$
\|\Phi\|_{a}(x ; X) \leq\|\Phi\|_{a} \chi_{\partial_{\Phi} X}(x),
$$

where we have used $\chi_{Y}$ to denote the characteristic function of a set $Y \subset \Lambda$, and we have introduced the $\Phi$-boundary of a set $X$, written $\partial_{\Phi} X$, given by

$$
\partial_{\Phi} X=\{x \in X: \exists Z \in S(X) \text { with } x \in Z \text { and } \Phi(Z) \neq 0\} .
$$

The Lieb-Robinson bound may be stated as follows. 
Theorem 2.1 (Lieb-Robinson Bound) Let $a \geq 0$ and take $\Phi \in \mathcal{B}_{a}(V)$. For any finite set $\Lambda \subset V$, denote by $\tau_{t}^{\Lambda}(\cdot)$ the time evolution corresponding to the local Hamiltonian

$$
H_{\Lambda}=\sum_{Z \subset \Lambda} \Phi(Z)
$$

Given any pair of local observables $A \in \mathcal{A}_{X}$ and $B \in \mathcal{A}_{Y}$ with $X, Y \subset \Lambda$ and $d(X, Y)>0$, one may estimate

$$
\left\|\left[\tau_{t}^{\Lambda}(A), B\right]\right\| \leq \frac{2\|A\|\|B\|}{\|\Phi\|_{a} C_{a}}\left(e^{2\|\Phi\|_{a} C_{a}|t|}-1\right) D_{a}(X, Y),
$$

for any $t \in \mathbb{R}$. Here the function $D_{a}(X, Y)$ is given by

$$
D_{a}(X, Y)=\min \left[\sum_{x \in X} \sum_{y \in Y}\|\Phi\|_{a}(x ; X) F_{a}(d(x, y)), \sum_{x \in X} \sum_{y \in Y}\|\Phi\|_{a}(y ; Y) F_{a}(d(x, y))\right] .
$$

A number of comments are useful in interpreting this theorem. First, we note that if $X$ and $Y$ have a non-empty intersection, then the argument provided below produces an analogous bound with the factor $e^{2\|\Phi\|_{a} C_{a}|t|}-1$ replaced by $e^{2\|\Phi\|_{a} C_{a}|t|}$. In the case of empty intersection and for small values of $|t|$, (13) is a better and sometimes useful estimates than the obvious bound $\left\|\left[\tau_{t}(A), B\right]\right\| \leq 2\|A\|\|B\|$, valid for all $t \in \mathbb{R}$.

Next, if $\Phi \in \mathcal{B}_{a}(V)$ for some $a>0$, then one has the trivial estimate that

$$
D_{a}(X, Y) \leq\left\|F_{0}\right\|\|\Phi\|_{a} \min \left(\left|\partial_{\Phi} X\right|,\left|\partial_{\Phi} Y\right|\right) e^{-a d(X, Y)} .
$$

Clearly then, we have that

$$
\left\|\left[\tau_{t}^{\Lambda}(A), B\right]\right\| \leq \frac{2\|A\|\|B\|\left\|F_{0}\right\|}{C_{a}} \min \left(\left|\partial_{\Phi} X\right|,\left|\partial_{\Phi} Y\right|\right) e^{-a\left[d(X, Y)-\frac{2\|\Phi\|_{a} C_{a}}{a}|t|\right]},
$$

which corresponds to a bound on the velocity of propagation given by

$$
v_{\Phi} \leq \inf _{a>0} \frac{2\|\Phi\|_{a} C_{a}}{a} .
$$

Next, we observe that for fixed local observables $A$ and $B$, the bounds above, (13) and (16), are independent of the volume $\Lambda \subset V$; given that $\Lambda$ contains the supports of both $A$ and $B$. Furthermore, we note that these bounds only require that one of the observables has finite support; in particular, if $|X|<\infty$ and $d(X, Y)>0$, then the bounds are valid irrespective of the support of $B$.

Proof of Theorem 2.1: To prove (13), we will provide an estimate on the quantity

$$
C_{B}(Z ; t)=\sup _{A \in \mathcal{A}_{Z}} \frac{\left\|\left[\tau_{t}^{\Lambda}(A), B\right]\right\|}{\|A\|},
$$

where $B$ is a fixed observable with support in $Y$, and the subset $Z \subset \Lambda$ we regard as arbitrary. Introduce the function

$$
f(t)=\left[\tau_{t}^{\Lambda}\left(\tau_{-t}^{X}(A)\right), B\right]
$$

where $A$ and $B$ are as in the statement of the theorem. Due to the strict locality of the Hamiltonian $H_{X}$, as defined e.g. in (12) and the fact that the observable $A \in \mathcal{A}_{X}$, we have that $\operatorname{supp}\left(\tau_{-t}^{X}(A)\right) \subset X$ for all $t \in \mathbb{R}$. It is straight forward to verify that

$$
f^{\prime}(t)=i \sum_{Z \in S_{\Lambda}(X)}\left[\tau_{t}^{\Lambda}(\Phi(Z)), f(t)\right]-i \sum_{Z \in S_{\Lambda}(X)}\left[\tau_{t}^{\Lambda}\left(\tau_{-t}^{X}(A)\right),\left[\tau_{t}^{\Lambda}(\Phi(Z)), B\right]\right] .
$$


Since the first term in (20) is norm preserving, we find that

$$
\left\|\left[\tau_{t}^{\Lambda}\left(\tau_{-t}^{X}(A)\right), B\right]\right\| \leq\|[A, B]\|+2\|A\| \sum_{Z \in S(X)} \int_{0}^{t}\left\|\left[\tau_{s}^{\Lambda}(\Phi(Z)), B\right]\right\| d s .
$$

The inequality (21) and the fact that $\left\|\tau_{-t}^{X}(A)\right\|=\|A\|$ together imply that

$$
C_{B}(X ; t) \leq C_{B}(X ; 0)+2 \sum_{Z \in S(X)}\|\Phi(Z)\| \int_{0}^{|t|} C_{B}(Z ; s) d s .
$$

It is clear from the definition, see (18), that for any finite $Z \subset \Lambda$,

$$
C_{B}(Z ; 0) \leq 2\|B\| \delta_{Y}(Z)
$$

where $\delta_{Y}(Z)=0$ if $Z \cap Y=\emptyset$ and $\delta_{Y}(Z)=1$ otherwise. Using this fact, iteration of (22) yields that

$$
C_{B}(X, t) \leq 2\|B\| \sum_{n=0}^{\infty} \frac{(2|t|)^{n}}{n !} a_{n}
$$

where for $n \geq 1$,

$$
a_{n}=\sum_{Z_{1} \in S(X)} \sum_{Z_{2} \in S\left(Z_{1}\right)} \cdots \sum_{Z_{n} \in S\left(Z_{n-1}\right)} \delta_{Y}\left(Z_{n}\right) \prod_{i=1}^{n}\left\|\Phi\left(Z_{i}\right)\right\| .
$$

For an interaction $\Phi \in \mathcal{B}_{a}(V)$, one may estimate that

$$
a_{1} \leq \sum_{x \in X} \sum_{y \in Y} \sum_{\substack{Z \in S(X): \\ x, y \in Z}}\|\Phi(Z)\| \leq \sum_{x \in X} \sum_{y \in Y}\|\Phi\|_{a}(x ; X) F_{a}(d(x, y)) .
$$

In addition,

$$
\begin{aligned}
a_{2} & \leq \sum_{x \in X} \sum_{y \in Y} \sum_{z \in \Lambda} \sum_{\substack{z_{1} \in S(X): \\
x, z \in Z_{1}}}\left\|\Phi\left(Z_{1}\right)\right\| \sum_{\substack{z_{2} \in S\left(Z_{1}\right): \\
z, y \in Z_{2}}}\left\|\Phi\left(Z_{2}\right)\right\| \\
& \leq\|\Phi\|_{a} \sum_{x \in X} \sum_{y \in Y} \sum_{z \in \Lambda} F_{a}(d(z, y)) \sum_{\substack{Z_{1} \in S(X): \\
x, z \in Z_{1}}}\left\|\Phi\left(Z_{1}\right)\right\| \\
& \leq\|\Phi\|_{a} \sum_{x \in X} \sum_{y \in Y} \sum_{z \in \Lambda} F_{a}(d(x, z)) F_{a}(d(z, y))\|\Phi\|_{a}(x ; X) \\
& \leq\|\Phi\|_{a} C_{a} \sum_{x \in X} \sum_{y \in Y}\|\Phi\|_{a}(x ; X) F_{a}(d(x, y)),
\end{aligned}
$$

where we have used (41) for the final inequality. With analogous arguments, one finds that for all $n \geq 1$,

$$
a_{n} \leq\left(\|\Phi\|_{a} C_{a}\right)^{n-1} \sum_{x \in X} \sum_{y \in Y}\|\Phi\|_{a}(x ; X) F_{a}(d(x, y)) .
$$

Inserting (28) into (24) we see that

$$
C_{B}(X, t) \leq \frac{2\|B\|}{\|\Phi\|_{a} C_{a}}\left(e^{2\|\Phi\|_{a} C_{a}|t|}-1\right) \sum_{x \in X} \sum_{y \in Y}\|\Phi\|_{a}(x ; X) F_{a}(d(x, y)),
$$

from which (13) immediately follows. 


\section{Quasi-locality of the dynamics}

The Lieb-Robinson bounds of Theorem 2.1 imply that the dynamics of quantum lattice systems, those generated by short range interactions, are quasi-local in the sense that the diameter of the support of any evolved local observable does not grow faster than linearly with time, up to an arbitrarily small error. There are at least two interesting ways to give precise meaning to this quasilocality property of the dynamics. In the first, one shows that the time-evolved observable can be well-approximated in norm by one with a strictly local support. This is achieved by the quantum version of integrating out the variables in the complement of a ball with a radius proportional to time. In the second, we show that to compute the dynamics up to a time $t>0$, one can replace the Hamiltonian with a local Hamiltonian supported in a ball of radius proportional to $t$. Clearly, the net result is the same: the support of observables evolved with approximate dynamics remains contained in the ball where the local Hamiltonian is supported. After presenting the details of these two approaches, we conclude this section with a few interesting applications that immediately follow from quasi-locality.

As in the previous section, we will work with an interaction $\Phi \in \mathcal{B}_{a}(V)$ with $a>0$. For the purpose of the discussion below, we will consider a finite subset $\Lambda \subset V$ and restrict our attention to the dynamics $\tau_{t}^{\Lambda}(\cdot)$ generated by the finite volume Hamiltonian $H_{\Lambda}$ as defined in (1); our bounds will be independent of the volume $\Lambda$. For any $X \subset \Lambda$ we will denote by $X^{c}=\Lambda \backslash X$.

In the first approach to obtaining a local approximation with support contained in $X \subset \Lambda$, one takes the normalized partial trace over the Hilbert space associated with $X^{c}$. In order to estimate the norm difference it is convenient to calculate the partial trace as an integral over the group of unitaries [5. Given an arbitrary observable $A \in \mathcal{A}_{\Lambda}$ and a set $X \subset \Lambda$, define

$$
\langle A\rangle_{X^{c}}=\int_{\mathcal{U}\left(X^{c}\right)} U^{*} A U \mu(d U),
$$

where $\mathcal{U}\left(X^{c}\right)$ denotes the group of unitary operators over the Hilbert space $\mathcal{H}_{X^{c}}$ and $\mu$ is the associated, normalized Haar measure. It is easy to see that for any $A \in \mathcal{A}_{\Lambda}$, the quantity $\langle A\rangle_{X^{c}}$ has been localized to $X$ in the sense that $\langle A\rangle_{X^{c}} \in \mathcal{A}_{X}$. Moreover, the difference may be written in terms of a commutator, i.e. as

$$
\langle A\rangle_{X^{c}}-A=\int_{\mathcal{U}\left(X^{c}\right)} U^{*}[A, U] \mu(d U) .
$$

To localize the dynamics, let $A \in \mathcal{A}_{X}$ and fix $\epsilon>0$. Based on our estimates in Theorem 2.1, we approximate the support of $\tau_{t}^{\Lambda}(A)$ with a time-dependent ball

$$
B_{t}(\epsilon, A)=\left\{x \in \Lambda: d(x, X) \leq \frac{2\|\Phi\|_{a} C_{a}}{a}|t|+\epsilon\right\} .
$$

For any unitary $U \in \mathcal{U}\left(B_{t}(\epsilon, A)^{c}\right)$, we clearly have that

$$
d(X, \operatorname{supp}(U)) \geq \frac{2\|\Phi\|_{a} C_{a}}{a}|t|+\epsilon
$$

and therefore, using (31) above and our bound (16), we immediately conclude that

$$
\begin{aligned}
\left\|\tau_{t}^{\Lambda}(A)-\left\langle\tau_{t}^{\Lambda}(A)\right\rangle_{B_{t}(\epsilon, A)^{c}}\right\| & \leq \int_{\mathcal{U}\left(B_{t}^{c}(\epsilon)\right)}\left\|\left[\tau_{t}^{\Lambda}(A), U\right]\right\| \mu(d U) \\
& \leq \frac{2\|A\|\left|\partial_{\Phi} X\right|}{C_{a}}\left\|F_{0}\right\| e^{-a \epsilon}
\end{aligned}
$$


The tolerance $\epsilon>0$ can be chosen to optimize estimates.

In the second approach, one shows that only those terms in the Hamiltonian supported in $B_{T}(\epsilon, A)$ contribute significantly to the time evolution of $A$ up to time $T$ [19]. Again, we consider the finite volume dynamics applied to a local observable $A \in \mathcal{A}_{X}$. Fix $\epsilon>0, T>0$, and consider the ball $B_{T}(\epsilon, A)$ as defined in (32) above. The estimate

$$
\left\|\tau_{t}^{\Lambda}(A)-\tau_{t}^{B_{T}(\epsilon, A)}(A)\right\| \leq \frac{\|A\|\left\|F_{0}\right\|\left|\partial_{\Phi} X\right|}{C_{a}^{2}}\left(C_{a}+\left\|F_{a}\right\|\right) e^{-a \epsilon}
$$

valid for all $|t| \leq T$, readily follows from the results in [19]. In fact, the proof of (35) uses the following basic estimate, see e.g. Lemma 3.3 in [19],

Lemma 3.1 Let $\Phi \in \mathcal{B}_{a}(V)$ with $a>0$ and take a finite subset $\Lambda \subset V$. If the finite volume Hamiltonian is written as the sum of two self-adjoint operators, $H_{\Lambda}=H_{\Lambda}^{(1)}+H_{\Lambda}^{(2)}$, and for $i=1,2$, $\tau_{t}^{(i)}(\cdot)$ denotes the dynamics corresponding to $H_{\Lambda}^{(i)}$, then the following estimate is valid:

$$
\left\|\tau_{t}^{\Lambda}(A)-\tau_{t}^{(1)}(A)\right\| \leq \int_{0}^{|t|}\left\|\left[H_{\Lambda}^{(2)}, \tau_{s}^{(1)}(A)\right]\right\| d s
$$

for any observable $A$ and $t \in \mathbb{R}$.

To apply Lemma 3.1 in the context discussed above, we write the local Hamiltonian as the sum of two terms:

$$
H_{\Lambda}=\sum_{\substack{Z \subset \Lambda: \\ Z \notin S_{\Lambda}\left(B_{T}(\epsilon, A)\right)}} \Phi(Z)+\sum_{\substack{Z \subset \Lambda: \\ Z \in S_{\Lambda}\left(B_{T}(\epsilon, A)\right)}} \Phi(Z)=H_{\Lambda}^{(1)}+H_{\Lambda}^{(2)} .
$$

Recall that for any $X \subset \Lambda$, we defined $S_{\Lambda}(X)$, the surface across $X$ in $\Lambda$, with equation (8) . Dropping the surface terms comprising $H_{\Lambda}^{(2)}$ above, decouples the dynamics, i.e., $\tau_{t}^{(1)}(A)=\tau_{t}^{B_{T}(\epsilon, A)}(A)$, and we find that for any $|t| \leq T$,

$$
\left\|\tau_{t}^{\Lambda}(A)-\tau_{t}^{B_{T}(\epsilon, A)}(A)\right\| \leq \sum_{\substack{Z \in \Lambda: \\ Z \in S_{\Lambda}\left(B_{T}(\epsilon, A)\right)}} \int_{0}^{|t|}\left\|\left[\Phi(Z), \tau_{s}^{B_{T}(\epsilon, A)}(A)\right]\right\| d s .
$$

For each of the terms on the right hand side above, the Lieb-Robinson estimates imply that

$$
\left\|\left[\Phi(Z), \tau_{s}^{B_{T}(\epsilon, A)}(A)\right]\right\| \leq \frac{2\|\Phi(Z)\|\|A\|}{C_{a}} e^{2\|\Phi\|_{a} C_{a}|s|} \sum_{z \in Z} \sum_{x \in \partial_{\Phi} X} F_{a}(d(x, z)) .
$$

Careful consideration of the combinatorics of these sums, exactly as done in [19], yields (35) as claimed.

It is interesting to ask about locality bounds for complex times. For one-dimensional systems with finite range interactions, Araki proved that the support does not grow faster than an exponential in $|z|$, where $z$ is the complex time [3, Theorem 4.2]. In other words, the complex time evolution

$$
\tau_{z}^{[-R, R]}(A)=e^{i z H_{\Lambda}} A e^{-i z H_{\Lambda}}
$$

can be approximated by $\tau_{z}^{[-N, N]}(A)$ with small error, uniformly in $R$, as long as $\log |z| \leq c N$, for a suitable constant $c$. For stochastic dynamics of classical particle systems, good locality bounds that are very similar to Theorem 2.1 are known [17, Theorem 4.20]. Since these classical models with 
stochastic dynamics can be thought of as quantum systems, generated by particular Hamiltonians and evolving with purely imaginary times, this may indicate that the general result, which allows for no more than logarithmic growth of $|z|$, should not be considered the final word on this issue in regards to specific models of interest.

The quasi-locality formulation of the Lieb-Robinson bounds makes it easy to derive bounds on double and higher order commutators of the form

$$
\left[\tau_{t_{1}}(A),\left[\tau_{t_{2}}(B), \tau_{t_{3}}(C]\right] .\right.
$$

Such commutators remain small in norm as long as $t_{1}, t_{2}$, and $t_{3}$ are such that the intersection of the time-dependent "quasi-supports" remains empty. That is whenever $t_{1}, t_{2}, t_{3} \in \mathbb{R}$ are such that

$$
B_{t_{1}}(\epsilon, A) \cap B_{t_{2}}(\epsilon, B) \cap B_{t_{3}}(\epsilon, C)=\emptyset .
$$

Another immediate application of the quasi-locality property is a bound on the rate at which spatial correlations can be created by the dynamics starting from a product state. In [19], we proved the following.

Theorem 3.2 Let $a>0, \Phi \in \mathcal{B}_{a}(V)$, and take $\Omega$ to be a normalized product state. Given $X, Y \subset \Lambda$ with $d(X, Y)>0$ and local observables $A \in \mathcal{A}_{X}$ and $B \in \mathcal{A}_{Y}$, the estimate

$$
\left|\left\langle\tau_{t}(A B)\right\rangle-\left\langle\tau_{t}(A)\right\rangle\left\langle\tau_{t}(B)\right\rangle\right| \leq 4\|A\|\|B\|\|F\|\left(\left|\partial_{\Phi} X\right|+\left|\partial_{\Phi} Y\right|\right) G_{a}(t) e^{-a d(X, Y)}
$$

is valid for all $t \in \mathbb{R}$. Here, for any observable $A$, the expectation value of $A$ in the state $\Omega$ is denoted by $\langle A\rangle=\langle\Omega, A \Omega\rangle$, and the function

$$
G_{a}(t)=\frac{C_{a}+\left\|F_{a}\right\|}{C_{a}}\|\Phi\|_{a} \int_{0}^{|t|} e^{2\|\Phi\|_{a} C_{a}|s|} d s .
$$

\section{Exponential Clustering}

As a second application of these locality bounds, we will present a proof of the Exponential Clustering Theorem, see Theorem 4.1 below, which improves on the estimates found in [21], see also [12. The proof of exponential clustering demonstrates that models with a spectral gap above the ground state energy necessarily exhibit exponential decay of spatial correlations in their ground state. Such results have recently appeared in a variety of contexts, e.g. [21, 12]. Using valence bond states, as is done in [18, one can easily construct gapless models with exponentially decaying ground state correlations indicating that, in general, there is no converse to Theorem 4.1 .

In the finite volume, the notion of a gapped Hamiltonian is clear. If the system is infinite, we express the gap condition in terms of the limiting dynamics, the existence of which is guaranteed by the Lieb-Robinson bounds as discussed above, by considering a representation of the system on a Hilbert space $\mathcal{H}$. This means that there is a representation $\pi: \mathcal{A}_{V} \rightarrow \mathcal{B}(\mathcal{H})$, and a self-adjoint operator $H$ on $\mathcal{H}$ such that

$$
\pi\left(\tau_{t}(A)\right)=e^{i t H} \pi(A) e^{-i t H}, \quad A \in \mathcal{A}_{V} .
$$

We will assume that the representative operator $H$ is non-negative and that there exists a vector $\Omega \in \mathcal{H}$ for which $H \Omega=0$. We say that the system has a spectral gap in the representation if there exists $\delta>0$ such that $\operatorname{spec}(H) \cap(0, \delta)=\emptyset$. In this case, the spectral gap, $\gamma$, is defined by

$$
\gamma=\sup \{\delta>0 \mid \operatorname{spec}(H) \cap(0, \delta)=\emptyset\} .
$$

Let $P_{0}$ denote the orthogonal projection onto ker $H$. From now on, we will work in this representation and simply write $A$ instead of $\pi(A)$. 
Theorem 4.1 (Exponential Clustering) Let $a>0$ and take $\Phi \in \mathcal{B}_{a}(V)$. Suppose that the dynamics corresponding to $\Phi$ on $V$ can be represented by a Hamiltonian $H$ with a gap $\gamma>0$ above the ground state energy, as described above. Let $\Omega$ be a normalized ground state vector for $H$; i.e. satisfy $H \Omega=0$ with $\|\Omega\|=1$. Then, there exists a constant $\mu>0$ such that for any local observables $A \in \mathcal{A}_{X}$ and $B \in \mathcal{A}_{Y}$ with $X, Y \subset V$ and $d(X, Y)>0$ satisfying $P_{0} B \Omega=P_{0} B^{*} \Omega=0$, the bound

$$
\left|\left\langle\Omega, A \tau_{i b}(B) \Omega\right\rangle\right| \leq C(A, B, \gamma) e^{-\mu d(X, Y)\left(1+\frac{\gamma^{2} b^{2}}{4 \mu^{2} d(X, Y)^{2}}\right)}
$$

is valid for all non-negative $b$ satisfying $0 \leq b \gamma \leq 2 \mu d(X, Y)$. One may take

$$
\mu=\frac{a \gamma}{4\|\Phi\|_{a} C_{a}+\gamma}
$$

as well as a constant

$$
C(A, B, \gamma)=\|A\|\|B\|\left[1+\sqrt{\frac{1}{\mu d(X, Y)}}+\frac{2\left\|F_{0}\right\|}{\pi C_{a}} \min \left(\left|\partial_{\Phi} X\right|,\left|\partial_{\Phi} Y\right|\right)\right] .
$$

Note that in the case of a non-degenerate ground state, the condition on $B$ is equivalent to $\langle\Omega, B \Omega\rangle=0$. In this case, the theorem with $b=0$ becomes

$$
|\langle\Omega, A B \Omega\rangle-\langle\Omega, A \Omega\rangle\langle\Omega, B \Omega\rangle| \leq C(A, B, \gamma) e^{-\mu d(X, Y)},
$$

which is the standard (equal-time) correlation function. For small $b>0$, the estimate (44) can be viewed as a perturbation of (47). Moreover, for $b>0$ large, there is a trivial bound

$$
\left|\left\langle\Omega, A \tau_{i b}(B) \Omega\right\rangle\right| \leq\|A\|\|B\| e^{-b \gamma} .
$$

Proof of Theorem 4.1:

We will follow very closely the proof which appears in [21] and refer to it whenever convenient. Consider the function $f$ given by

$$
f(z)=\left\langle\Omega, A \tau_{z}(B) \Omega\right\rangle=\int_{\gamma}^{\infty} e^{i z E} d\left\langle A^{*} \Omega, P_{E} B \Omega\right\rangle
$$

where we have used the spectral theorem and the fact that $B$ projects off the ground state. It is clear that the function $f$ defined in (49) is analytic in the upper half plane and has a continuous (and bounded) boundary value on the real axis. The quantity we wish to bound corresponds to $f(i b)$ for $b>0$. The case $b=0$ will follow by a limiting argument.

Since the boundary value of $f$ on $\mathbb{R}$ is continuous, one may show by a limiting argument that for any $T>b$,

$$
f(i b)=\frac{1}{2 \pi i} \int_{\Gamma_{T}} \frac{f(z)}{z-i b} d z
$$

where $\Gamma_{T}$ is the semi-circular contour from $-T$ to $T$ (on the real axis) into the upper half plane. As is shown in [21], the fact that the Hamiltonian is gapped, see also (49), implies that the integral over the circular part of the contour vanishes in the limit as $T \rightarrow \infty$, and therefore we have the bound

$$
\left|\left\langle\Omega, A \tau_{i b}(B) \Omega\right\rangle\right|=|f(i b)| \leq \limsup _{T \rightarrow \infty}\left|\frac{1}{2 \pi i} \int_{-T}^{T} \frac{f(t)}{t-i b} d t\right|
$$


The proof is then complete once we estimate this integral over the real line. While this inequality is true for any value of $b>0$, to get the desired estimate, we will have to choose $b>0$ sufficiently small; see the comments following (64) below.

Let $\alpha>0$. We will choose this free parameter later. Observe that one may write

$$
f(t)=e^{-\alpha b^{2}}\left[f(t) e^{-\alpha t^{2}}+f(t)\left(e^{\alpha b^{2}}-e^{-\alpha t^{2}}\right)\right] .
$$

Clearly then, the integral we wish to bound, the one on the right hand side of (51) above, can be estimated by

$$
e^{-\alpha b^{2}}\left|\frac{1}{2 \pi i} \int_{-T}^{T} \frac{f(t) e^{-\alpha t^{2}}}{t-i b} d t\right|+e^{-\alpha b^{2}}\left|\frac{1}{2 \pi i} \int_{-T}^{T} \frac{f(t)\left(e^{\alpha b^{2}}-e^{-\alpha t^{2}}\right)}{t-i b} d t\right| .
$$

We will bound the absolute value of each of the integrals appearing in (53) separately; the prefactor $e^{-\alpha b^{2}}$ will be an additional damping made explicit by the choice of $\alpha$.

To bound the first integral appearing in (153), we further divide the integrand into two terms. Note that

$$
f(t) e^{-\alpha t^{2}}=\left\langle\Omega, \tau_{t}(B) A \Omega\right\rangle e^{-\alpha t^{2}}+\left\langle\Omega,\left[A, \tau_{t}(B)\right] \Omega\right\rangle e^{-\alpha t^{2}} .
$$

By the spectral theorem, we have that

$$
\frac{1}{2 \pi i} \int_{-T}^{T} \frac{\left\langle\Omega, \tau_{t}(B) A \Omega\right\rangle e^{-\alpha t^{2}}}{t-i b} d t=\int_{\gamma}^{\infty} \frac{1}{2 \pi i} \int_{-T}^{T} \frac{e^{-i t E} e^{-\alpha t^{2}}}{t-i b} d t d\left\langle P_{E} B^{*} \Omega, A \Omega\right\rangle,
$$

where we have used now that $B^{*} \Omega$ is also orthogonal to the ground state. Applying Lemma 4.2 , stated below, to the inner integral above, we have that

$$
\lim _{T \rightarrow \infty} \frac{1}{2 \pi i} \int_{-T}^{T} \frac{e^{-i t E} e^{-\alpha t^{2}}}{t-i b} d t=\frac{1}{2 \sqrt{\pi \alpha}} \int_{0}^{\infty} e^{-b w} e^{-\frac{(w+E)^{2}}{4 \alpha}} d w \leq \frac{1}{2} e^{-\frac{\gamma^{2}}{4 \alpha}}
$$

where for the final inequality above we used that $E \geq \gamma>0, \alpha>0$, and $b>0$. From this we easily conclude that

$$
\limsup _{T \rightarrow \infty}\left|\frac{1}{2 \pi i} \int_{-T}^{T} \frac{\left\langle\Omega, \tau_{t}(B) A \Omega\right\rangle e^{-\alpha t^{2}}}{t-i b} d t\right| \leq \frac{\|A\|\|B\|}{2} e^{-\frac{\gamma^{2}}{4 \alpha}}
$$

For the integral corresponding to the second term in (54), it is easy to see that

$$
\left|\frac{1}{2 \pi i} \int_{-T}^{T} \frac{\left\langle\Omega,\left[A, \tau_{t}(B)\right] \Omega\right\rangle e^{-\alpha t^{2}}}{t-i b} d t\right| \leq \frac{1}{2 \pi} \int_{-\infty}^{\infty} \frac{\left\|\left[A, \tau_{t}(B)\right]\right\|}{|t|} e^{-\alpha t^{2}} d t
$$

where we have taken advantage of the fact that $b>0$. To complete our estimate, we will introduce another free parameter $s>0$. Here we use the Lieb-Robinson bound, Theorem 2.1, for times $|t| \leq s$ and a basic norm estimate otherwise. The result is that the right hand side of (58) is bounded from above by

$$
\frac{2\|A\|\|B\|}{\pi\|\Phi\|_{a} C_{a}} D_{a}(X, Y)\left(e^{2\|\Phi\|_{a} C_{a} s}-1\right)+\frac{\|A\|\|B\|}{s \sqrt{\pi \alpha}} e^{-\alpha s^{2}} .
$$

This completes the bound of the first integral appearing in (53). 
Using again the spectral theorem, the second integral in (53) may be written as

$$
\left|\int_{\gamma}^{\infty} \frac{1}{2 \pi i} \int_{-T}^{T} \frac{e^{i t E}\left(e^{\alpha b^{2}}-e^{-\alpha t^{2}}\right)}{t-i b} d t d\left\langle A^{*} \Omega, P_{E} B \Omega\right\rangle\right| .
$$

As is described in detail in [21], we find that for $E \geq \gamma$ and $\alpha$ chosen such that $\gamma \geq 2 \alpha b$,

$$
\lim _{T \rightarrow \infty} \frac{1}{2 \pi i} \int_{-T}^{T} \frac{e^{i t E}}{t-i b}\left(e^{\alpha b^{2}}-e^{-\alpha t^{2}}\right) d t \leq \frac{1}{2} e^{-\frac{\gamma^{2}}{4 \alpha}}
$$

which produces an estimate (one analogous to the bound in (57) above) for (60).

All of our estimates above combine to demonstrate that the right hand side of (51) is bounded by

$$
\|A\|\|B\|\left[e^{-\frac{\gamma^{2}}{4 \alpha}}+\frac{2 D_{a}(X, Y)}{\pi\|\Phi\|_{a} C_{a}}\left(e^{2\|\Phi\|_{a} C_{a} s}-1\right)+\frac{1}{s \sqrt{\pi \alpha}} e^{-\alpha s^{2}}\right]
$$

if $\alpha$ satisfies $\gamma \geq 2 \alpha b$. The choice $\alpha=\gamma / 2 s$ yields:

$$
\|A\|\|B\| e^{-\frac{\gamma s}{2}}\left[1+\sqrt{\frac{2}{\pi \gamma s}}+\frac{2 D_{a}(X, Y)}{\pi\|\Phi\|_{a} C_{a}} e^{\left(2\|\Phi\|_{a} C_{a}+\frac{\gamma}{2}\right) s}\right]
$$

As is demonstrated in (15), $D_{a}(X, Y)$ decays exponentially as $e^{-a d(X, Y)}$. In this case, if we choose $s$ to be the solution of the equation

$$
s\left(2\|\Phi\|_{a} C_{a}+\gamma / 2\right)=\operatorname{ad}(X, Y),
$$

then we have proven the result. Notice that we have chosen $\alpha$ in terms of $s$, which is defined independently of $b$, thus the condition $\gamma \geq 2 \alpha b$ will be satisfied for sufficiently small $b>0$.

In our proof of the Exponential Clustering Theorem above, we used several times the following useful fact, a proof of which appears in [21].

Lemma 4.2 Let $E \in \mathbb{R}, \alpha>0$, and $z \in \mathbb{C}^{+}=\{z \in \mathbb{C}: \operatorname{Im}[z]>0\}$. One has that

$$
\lim _{T \rightarrow \infty} \frac{1}{2 \pi i} \int_{-T}^{T} \frac{e^{i E t} e^{-\alpha t^{2}}}{t-z} d t=\frac{1}{2 \sqrt{\pi \alpha}} \int_{0}^{\infty} e^{i w z} e^{-\frac{(w-E)^{2}}{4 \alpha}} d w .
$$

Moreover, the convergence is uniform for $z \in \mathbb{C}^{+}$.

\section{The Lieb-Schultz-Mattis Theorem}

As a final application of these locality bounds, in particular both Theorem 2.1 and Theorem 4.1 . we were recently able to provide a rigorous proof the Lieb-Schultz-Mattis theorem, see [23], which is valid in arbitrary dimensions. In this section, we will discuss this result and outline the ideas which motivate our proof. 


\subsection{The Result and Some Words on the Proof}

The classical Lieb-Schultz-Mattis Theorem (LSM), 15], concerns the spin-1/2, anti-ferromagnetic Heisenberg chain. This model is defined through a family of Hamiltonians $H_{L}$, acting on the Hilbert space $\mathcal{H}_{[1, L]}=\bigotimes_{x \in[1, L]} \mathbb{C}^{2}$, with the form

$$
H_{L}=\sum_{x=1}^{L-1} \boldsymbol{S}_{x} \cdot \boldsymbol{S}_{x+1} .
$$

Here, for each integer $x \in[1, L]$, the spin vectors $\boldsymbol{S}_{x}$ have components

$$
S_{x}^{j}=\mathbb{1} \otimes \cdots \otimes \mathbb{1} \otimes S^{j} \otimes \mathbb{1} \otimes \cdots \otimes \mathbb{1}, \quad j=1,2,3,
$$

where $S^{j}$ is the corresponding spin-1/2 (Pauli) matrix acting on the $x$-th factor of $\mathcal{H}_{[1, L]}$. The LSM Theorem may be stated as follows.

Theorem 5.1 (LSM, 1961) If the ground state of $H_{L}$ is unique, then the gap in energy between the ground state and the first excited state is bounded by $C / L$.

A further result by Lieb and Mattis in 1966, [14, verified that under certain conditions, for example when $L$ is even, the main assumption in the LSM Theorem, specifically the uniqueness of the ground state, is indeed satisfied. Almost twenty years later, the LSM Theorem was generalized to encompass a variety of other one (and quasi-one) dimensional models by Affleck and Lieb in [1. In particular, this result applies to those chains of even length with spins having arbitrary halfinteger magnitude. Note: Here and in the rest of this section the term half-integer, or half-integral, refers to one-half of a positive odd integer, i.e., an element of the set $\mathbb{N}+1 / 2$.

For models to which the LSM Theorem applies, one expects that the excitation spectrum corresponding to the thermodynamic limit has no gap above the ground state energy. It is interesting to note that the predictions of Haldane [9] suggest that such a result is rather sensitive to the type of interaction terms. In fact, the spin-1, anti-ferromagnetic Heisenberg chain is predicted to have a robust gap above the ground state energy in the thermodynamic limit.

In a work of 2004, see [10], Hastings argued that a higher dimensional analogue of the LSM Theorem could be proven using the improved locality bounds which have recently been established. We will now summarize these ideas and indicate how they may be implemented to demonstrate a rigorous proof of this theorem.

The multi-dimensional LSM Theorem, stated as Theorem 5.2 below, is valid for a large class of models; a detailed proof of this is contained in [23]. For simplicity of presentation in this review article, we will restrict our attention to the spin-1/2, Heisenberg anti-ferromagnet, however, our general assumptions are discussed in Subsection 5.2.4. In $\nu$ dimensions, the model of interest is defined on subsets $V_{L} \subset \mathbb{Z}^{\nu}$ in analogy to (66) above, i.e. one considers Hamiltonians

$$
H_{L}=\sum_{\substack{x, y \in V_{L} \\|x-y|=1}} \boldsymbol{S}_{x} \cdot \boldsymbol{S}_{y}
$$

acting on the Hilbert space $\mathcal{H}_{V_{L}}=\bigotimes_{x \in V_{L}} \mathbb{C}^{2}$. It is easy to state the new result.

Theorem 5.2 If the ground state of $H_{L}$ is non-degenerate, then the gap, $\gamma_{L}$, above the ground state energy satisfies

$$
\gamma_{L} \leq C \frac{\log (L)}{L} .
$$


The logarthmic correction which appears in Theorem 5.2, in contrast to the original result of Theorem 5.1, seems to be an inevitable consequence of the locality bounds we incorporate in our proof. It is an interesting open question to determine whether or not there is a class of models, in dimensions $\nu>1$, for which one can prove such a bound without the logarithmic correction.

In essense, Theorem 5.2 is proven using a variational argument. Letting $\psi_{0}$ denote the unique, normalized ground state, we know that for any normalized vector $\psi_{1}$ with $\left|\left\langle\psi_{0}, \psi_{1}\right\rangle\right| \neq 1$, the bound

$$
0<\gamma \leq \frac{\left\langle\psi_{1},\left(H-E_{0}\right) \psi_{1}\right\rangle}{1-\left|\left\langle\psi_{0}, \psi_{1}\right\rangle\right|^{2}}
$$

is always valid. Here we have dropped the dependence of all quantities on the length scale $L$. From this perspective, there are only three steps necessary to prove the desired result. First, we must construct a normalized trial state $\psi_{1}$, as indicated above. Next, we must estimate the difference in the energy corresponding to $\psi_{1}$ and $E_{0}$. Lastly, we must ensure that the inner product $\left|\left\langle\psi_{0}, \psi_{1}\right\rangle\right|$ remains sufficiently small.

This method of proof is complicated by the fact that the ground state is virtually unknown, and therefore the means by which one should construct a trial state is not apriori clear. Inspiration for the construction of our variational state comes from the work of Hastings, again see [10], in which he proposes to consider the ground state of a modified Hamiltonian, $H_{\theta}$, where the interactions in a given hyperplane have been twisted by an angle of $\theta$; more on this below. The ground state of this modified Hamiltonian may be regarded as the solution of a specific differential equation, in the variable $\theta$, whose initial condition corresponds to the unique ground state whose existence we assumed. The solution of Hastings' differential equation is ameanable to analysis, in particular, one can apply both the Lieb-Robinson bounds and the Exponential Clustering Theorem to provide the desired energy and orthogonality estimates mentioned above. One may recall that the clustering bounds, as in Theorem 4.1, provide estimates which themselves depend on the size of gap $\gamma_{L}$. For this reason, the argument proceeds by way of contradiction. In fact, by assuming that there exists a sufficiently large constant $C$ for which the gap satisfies $\gamma_{L}>C \log (L) / L$ for large enough $L$, we construct a trial state whose energy eventually violates this bound.

\subsection{A More Detailed Outline of the Proof}

\subsubsection{Constructing the Trial State}

In our proof, we use the fact that the Hamiltonians we consider are assumed to have at least one direction of translation invariance. We incorporate this into our notation by considering finite subsets $V_{L} \subset \mathbb{Z}^{\nu}$ of the form $V_{L}=[1, L] \times V_{L}^{\perp}$ where we have isolated a particular direction, which we will often refer to as the horizontal direction, and perpendicular sets $V_{L}^{\perp} \subset \mathbb{Z}^{\nu-1}$ with cardinality $\left|V_{L}^{\perp}\right| \leq C L^{\nu-1}$. For the orthogonality result, we will also need to assume that $\left|V_{L}^{\perp}\right|$ is odd, see Subsection 5.2.3. The trial state is constructed from a perturbation of the Hamiltonian $H_{L}$ defined by "twisting" certain interaction terms. A twist in the hyperplane situated at a site $m \in[1, L]$ is defined by replacing all interaction terms $\boldsymbol{S}_{x} \cdot \boldsymbol{S}_{y}$ in (68) corresponding to horizontal bonds with $x=(m, v), y=(m+1, v)$, and some $v \in V_{L}^{\perp}$ by terms of the form

$$
h_{x y}(\theta)=\boldsymbol{S}_{x} \cdot e^{-i \theta S_{y}^{3}} \boldsymbol{S}_{y} e^{i \theta S_{y}^{3}}
$$

for some $\theta \in \mathbb{R}$. A doubly twisted Heisenberg Hamiltonian is then given by

$$
H_{\theta, \theta^{\prime}}=\sum_{\substack{x, y \in V_{L}: \\|x-y|=1}} h_{x y}\left(\theta_{x y}\right)
$$



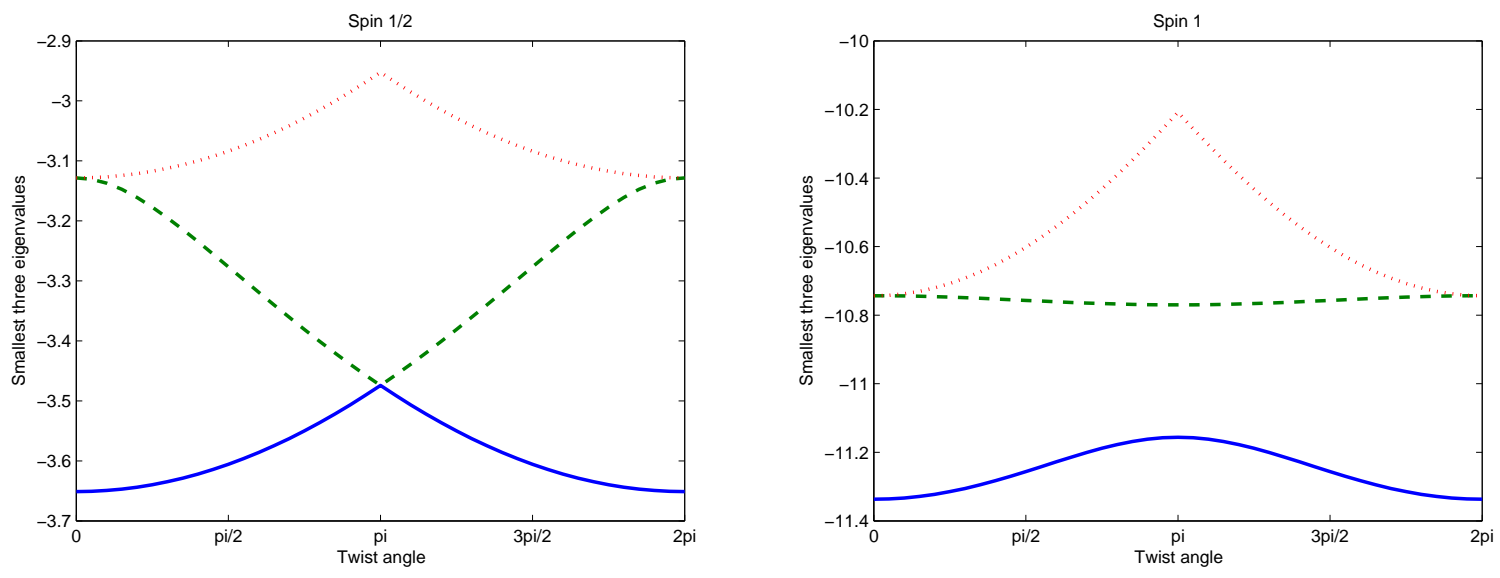

Figure 1: Plot of the three lowest eigenvalues of $H_{\theta, 0}$, the singly twisted Heisenberg Hamiltonian for a ring of 8 spins. The magnitude of spins is $S=1 / 2$ in the plot on the left and $S=1$ on the right.

where

$$
\theta_{x y}= \begin{cases}\theta, & \text { if } x=(m, v), y=(m+1, v) \text { for some } v \in V_{L}^{\perp}, \\ \theta^{\prime}, & \text { if } x=(m+L / 2, v), y=(m+1+L / 2, v) \text { for } v \in V_{L}^{\perp}, \\ 0, & \text { otherwise. }\end{cases}
$$

Here we have taken periodic boundary conditions in the horizontal direction.

It is interesting to note the behavior of the lowest eigenvalues of the singly twisted Heisenberg Hamiltonian $H_{\theta, 0}$ for a simple spin ring with an even number of spins. The behavior depends in an interesting way on the magnitude of the spins. When the spins are half-integer, the two lowest eigenvalues cross at $\theta=\pi$. In contrast, when the spins are integer, they remain non-degenerate. The quasi-adiabatic evolution is a device designed to construct a continuous path from the ground state of $H_{0,0}$ to the first excited state of $H_{2 \pi, 0}$ which, of course, are both identical to the unperturbed Hamiltonian.

One of Hastings' crucial insights in [10] is that, for the half-integer spin case, the first excited state can be obtained by applying a "quasi-adiabatic evolution" to the ground state; here $\theta$ is the evolution parameter. More concretely, let $\psi_{0}\left(\theta, \theta^{\prime}\right)$ and $E_{0}\left(\theta, \theta^{\prime}\right)$ denote the ground state and ground state energy of the doubly twisted Hamiltonian $H_{\theta, \theta^{\prime}}$, respectively. It is easy to see that along the path $\theta^{\prime}=-\theta$, the Hamiltonian $H_{\theta,-\theta}$ is unitarily equivalent to the unperturbed Hamiltonian $H_{L}=H_{0,0}$. By differentiating the eigenvalue equation $H_{\theta,-\theta} \psi_{0}(\theta,-\theta)=E_{0}(\theta,-\theta) \psi_{0}(\theta,-\theta)$ and using that $\partial_{\theta} E_{0}(\theta,-\theta)=0$, one obtains

$$
\partial_{\theta} \psi_{0}(\theta,-\theta)=-\frac{1}{H_{\theta,-\theta}-E_{0}}\left[\partial_{\theta} H_{\theta,-\theta}\right] \psi_{0}(\theta,-\theta) .
$$

Formally, equation (174) may be re-written using the Heisenberg dynamics as follows,

$$
\partial_{\theta} \psi_{0}(\theta,-\theta)=B(\theta) \psi_{0}(\theta,-\theta),
$$

where the operator $B(\theta)$ is defined by

$$
B(\theta)=-\int_{0}^{\infty} \tau_{i t}\left(\partial_{\theta} H_{\theta,-\theta}\right) P_{0}(\theta,-\theta) d t .
$$


Here we have denoted by $\tau_{t}(\cdot)$ the dynamics generated by the Hamiltonian $H_{\theta,-\theta}$, and $P_{0}(\theta,-\theta)$ is the corresponding spectral projection onto the ground state. Due to the gap assumption, (76) is well-defined.

Equation (75) captures the evolution of the ground state of a doubly twisted Hamiltonian along the path $\theta^{\prime}=-\theta$ where the effect of the first twist is canceled by the second. The trial state is obtained by using an approximation of the differential equation in (75) to describe the ground state of the singly twisted Hamiltonian $H_{\theta, 0}$. Introduce the parameters $\alpha>0$ and $T>0$. To approximate the imaginary time evolution corresponding to an arbitrary Hamiltonian $H$, with dynamics $\tau_{s}(\cdot)$, at time $t>0$, we define

$$
A_{\alpha}(i t, H)=\frac{1}{2 \pi i} \int_{-\infty}^{+\infty} \tau_{s}(A) \frac{e^{-\alpha s^{2}}}{s-i t} d s
$$

for any observable $A$. In general, an anti-Hermitian operator of the form

$$
B_{\alpha, T}(A, H)=-\int_{0}^{T}\left[A_{\alpha}(i t, H)-A_{\alpha}(i t, H)^{*}\right] d t,
$$

will be used in place of (76) .

Note that the observable which is evolved in equation (76), $\partial_{\theta} H_{\theta,-\theta}$, contains terms of two types. The first are localized around the twist of angle $\theta$ which correspond to horizontal bonds $(x, y)$ of the type $x=(m, v), y=(m+1, v)$, and some $v \in V_{L}^{\perp}$. The second are similar, yet localized around the twist of angle $-\theta$. We group these two types of terms together and write $\partial_{\theta} H_{\theta,-\theta}=\partial_{1} H_{\theta,-\theta}-\partial_{2} H_{\theta,-\theta}$ to simplify notation. By linearity, the operator

$$
B_{\alpha, T}\left(\partial_{\theta} H_{\theta,-\theta}, H_{\theta,-\theta}\right)=B_{\alpha, T}\left(\partial_{1} H_{\theta,-\theta}, H_{\theta,-\theta}\right)-B_{\alpha, T}\left(\partial_{2} H_{\theta,-\theta}, H_{\theta,-\theta}\right) .
$$

Given a sufficiently large gap $\gamma_{L}$, there is a choice of the parameters $\alpha$ and $T$ for which the ground state $\psi_{0}(\theta,-\theta)$ is well approximated by the solution of the differential equation (75) with $B(\theta)$ replaced by $B_{\alpha, T}\left(\partial_{\theta} H_{\theta,-\theta}, H_{\theta,-\theta}\right)$. Hastings' clever proposal is that one may also approximate the ground state of the singly twisted Hamiltonian, $\psi_{0}(\theta, 0)$, by evolving with just the first term on the right hand side of (79) above. More concretely, consider the operator $B_{\alpha, T}(\theta)$ defined by setting $B_{\alpha, T}(\theta)=B_{\alpha, T}\left(\partial_{1} H_{\theta,-\theta}, H_{\theta,-\theta}\right)$ and solve the Hastings' Equation given by

$$
\partial_{\theta} \psi_{\alpha, T}(\theta)=B_{\alpha, T}(\theta) \psi_{\alpha, T}(\theta)
$$

with initial condition $\psi_{\alpha, T}(0)=\psi_{0}(0,0)$, i.e., the ground state of the unperturbed Hamiltonian. Under the choice of parameters $\alpha=\gamma_{L} / L$ and $T=L / 2$, the resulting variational state $\psi_{1}=$ $\psi_{\alpha, T}(2 \pi)$ may be estimated in such a way that Theorem 5.2 follows. We note that a particularly nice feature of the differential equation (80) is that the operator $B_{\alpha, T}(\theta)$ is anti-Hermitian, and therefore the solution remains normalized for all $\theta \in[0,2 \pi]$. Hastings interprets the solution of (80) as a quasi-adiabatic evolution of the ground state $\psi_{0}=\psi_{0}(0,0)$.

\subsubsection{Locality and the Trial State}

One key technical lemma, which we use repeatedly in all of the estimates that follow, concerns the local evolution of the solution to (80). Consider a sub-volume $\Lambda_{L}(m) \subset V_{L}$ localized around the twist of angle $\theta$, for example, take $\Lambda_{L}(m)$ to be of the form $[m-(L / 4-2), m+(L / 4-2)] \times V_{L}^{\perp}$ which is slightly less than half of the system. Let $\rho_{\alpha, T}(\theta)$ and $\rho_{0}(\theta,-\theta)$ denote the density matrices corresponding to the states $\psi_{\alpha, T}(\theta)$ and $\psi_{0}(\theta,-\theta)$. 
Lemma 5.3 Suppose there exists a constant $c>0$ such that $L \gamma_{L} \geq c$ and choose $\alpha=\gamma_{L} / L$ and $T=L / 2$. Then, there exists constants $C>0$ and $k>0$ such that

$$
\sup _{\theta \in[0,2 \pi]}\left\|\operatorname{Tr}_{V_{L} \backslash \Lambda_{L}(m)}\left[\rho_{\alpha, T}(\theta)-\rho_{0}(\theta,-\theta)\right]\right\|_{1} \leq C L^{2 \nu} e^{-k L \gamma_{L}} .
$$

Since the proof proceeds by way of contradiction, the assumption $L \gamma_{L} \geq c$ is part of the argument. Moreover, it is easy to produce a bound on $\gamma_{L}$ from above that is independent of the length scale $L$. Lemma 5.3 demonstrates that if $L \gamma_{L}$ is sufficiently large, then the effect of ignoring the second twist in the definition of $\psi_{\alpha, T}(\theta)$ is negligible when one restricts their attention to observables localized in $\Lambda_{L}(m)$. The proof of this lemma uses both the Lieb-Robinson bound, Theorem 2.1, and the Exponential Clustering result, Theorem 4.1.

\subsubsection{The Estimates}

The Energy Estimate: To estimate the energy of the trial state, we consider the function

$$
E(\theta)=\left\langle\psi_{\alpha, T}(\theta), H_{\theta,-\theta} \psi_{\alpha, T}(\theta)\right\rangle
$$

Due to the initial condition used to define $\psi_{\alpha, T}(\theta)$, we know that $E(0)=E_{0}$ the ground state energy, and since $H_{2 \pi,-2 \pi}=H_{L}, E(2 \pi)$ corresponds to the energy of the trial state $\psi_{1}=\psi_{\alpha, T}(2 \pi)$. The main idea here is to use the locality property of the trial state, i.e. Lemma 5.3, and the unitary equivalence of the Hamiltonians $H_{\theta,-\theta}$ to obtain an estimate on the derivative of this function which is uniform for $\theta \in[0,2 \pi]$. Explicitly, we can prove the following result.

Theorem 5.4 Suppose there exists a constant $c>0$ such that $L \gamma_{L} \geq c$ and choose $\alpha=\gamma_{L} / L$ and $T=L / 2$. Then, there exists constants $C>0$ and $k>0$ such that

$$
\left|\left\langle\psi_{1}, H_{L} \psi_{1}\right\rangle-E_{0}\right| \leq C L^{3 \nu-1} e^{-k L \gamma_{L}} .
$$

The Orthogonality Estimate: As we mentioned before, Hastings' quasi-adiabatic evolution is norm preserving. In particular, we are guaranteed that $\left\|\psi_{1}\right\|=\left\|\psi_{0}\right\|=1$. Our argument that $\psi_{1}$ is sufficiently orthogonal to $\psi_{0}$ makes essential use of the fact that the total spin in each perpendicular set, $V_{L}^{\perp}$, is half-integer. In the case of the spin-1/2, anti-ferromagnetic Heisenberg model, this corresponds to the assumption that $\left|V_{L}^{\perp}\right|$ is odd. We have the following theorem.

Theorem 5.5 Suppose there exists a constant $c>0$ such that $L \gamma_{L} \geq c$ and choose $\alpha=\gamma_{L} / L$ and $T=L / 2$. Then, there exists constants $C>0$ and $k>0$ such that

$$
\left|\left\langle\psi_{1}, \psi_{0}\right\rangle\right| \leq C L^{2 \nu} e^{-k L \gamma_{L}}
$$

To prove this result we observe that, although the ground state $\psi_{0}(\theta,-\theta)$ of the perturbed Hamiltonian is not translation invariant, it is invariant with respect to "twisted" translations. In fact, let $T$ be a unitary implementing the translation symmetry in the horizontal direction, specifically the direction in which we have imposed periodic boundary conditions, chosen such that $T \psi_{0}=\psi_{0}$. This is possible since $\psi_{0}$ is the unique ground state and the Hamiltonian is translation invariant with respect to $T$, i.e., $T^{*} H_{L} T=H_{L}$.

Define twisted translations by setting

$$
T_{\theta, \theta^{\prime}}=T U_{m}(\theta) U_{m+L / 2}\left(\theta^{\prime}\right)
$$


where the column rotation, $U_{n}(\theta)$, applies the rotation $e^{i \theta S_{x}^{3}}$ to all sites for the form $x=(n, v)$, for some $v \in V_{L}^{\perp}$. The unitary equivalence of the doubly twisted Hamiltonian $H_{\theta,-\theta}$ to $H_{L}=H_{0,0}$ can also be expressed in terms of these column rotations

$$
H_{\theta,-\theta}=W(\theta)^{*} H_{0,0} W(\theta)
$$

where

$$
W(\theta)=\bigotimes_{m<n \leq m+L / 2} U_{n}(\theta) .
$$

With these definitions, it is easy to see that $W(\theta)^{*} T W(\theta)=T_{\theta,-\theta}$ commutes with $H_{\theta,-\theta}$, and therefore,

$$
T_{\theta,-\theta} \psi_{0}(\theta,-\theta)=\psi_{0}(\theta,-\theta)
$$

as we claimed.

The main idea in the proof of Theorem 5.5 is to again use the locality properties of the solution of Hastings' Equation (80) to show that

$$
T_{\theta, 0} \psi_{\alpha, T}(\theta) \sim \psi_{\alpha, T}(\theta)
$$

Since the total spin in each $V_{L}^{\perp}$ is half-integer, again a consequence of assuming $\left|V_{L}^{\perp}\right|$ is odd, the column rotation $U_{m}(2 \pi)=-1$. Clearly then, $T_{2 \pi, 0}=-T$, and therefore, $T \psi_{1} \sim-\psi_{1}$. As we have chosen $T$ so that, $T \psi_{0}=\psi_{0}$, this implies that $\psi_{1}$ is nearly orthogonal to $\psi_{0}$.

\subsubsection{Generalizations}

As we previously indicated, the proof of the multi-dimensional Lieb-Schultz-Mattis Theorem, demonstrated in [23], applies to a large variety of models. In what follows below, we will outline a list of assumptions which define a wide class of Hamiltonians for which the LSM Theorem remains valid.

The Basic Set-Up: It is not important for our argument that the underlying sets have a lattice structure, in particular the sets $V_{L}$ need not be subsets of $\mathbb{Z}^{\nu}$. Rather, we need only assume that there is, at least, one direction of increase, which previously we labeled the horizontal direction. We make this notion concrete by assuming that there exists an increasing sequence of finite sets $\left\{V_{L}\right\}_{L \geq 1}$, exhausting some infinite set $V$, which are of the form $V_{L}=[1, L] \times V_{L}^{\perp}$. We also assume a bound on the cardinality of the perpendicular sets of the form $\left|V_{L}^{\perp}\right| \leq c L^{\alpha}$ for some $\alpha \geq 0$, and it is natural, but not necessary, to take $\alpha=\nu-1$.

The interactions can also be of a general form. We assume that the set $V$ is equipped with a metric $d$ and a function $F$ as described in Section 2, To start with, we work with interactions $\Phi \in \mathcal{B}_{a}(V)$ for some $a>0$ so that the infinite volume dynamics is well defined from the beginning. In contrast to (68), the more general finite volume Hamiltonians are of the form

$$
H_{L}=\sum_{X \subset V_{L}} \Phi(X)+\text { boundary terms, }
$$

where we will assume periodic boundary conditions in the horizontal direction and arbitrary boundary conditions in the other directions.

Assumption I. Our first assumption is that the interaction $\Phi \in \mathcal{B}_{a}(V)$ is translation invariant in the horizontal direction. This is clearly the case for the Heisenberg anti-ferromagnet, and in general, it means that for any $X \subset V_{L}$,

$$
\Phi\left(X+e_{1}\right)=\alpha_{1}(\Phi(X)),
$$


where $X+e_{1}$ is the translation of all points in $X$ by one unit in the horizontal direction and $\alpha_{1}(\cdot)$ is the translation automorphism which maps $\mathcal{A}_{\left(n, V_{L}^{\perp}\right)}$ into $\mathcal{A}_{\left(n+1, V_{L}^{\perp}\right)}$ for all $n \in \mathbb{Z}$. Here the column sets $\left(n, V_{L}^{\perp}\right)$ are defined by $\left(n, V_{L}^{\perp}\right)=\left\{x \in V_{L}: x=(n, v)\right.$ for some $\left.v \in V_{L}^{\perp}\right\}$. Due to the assumed periodicity in the horizontal direction, this translation invariance can be implemented by a unitary $T \in \mathcal{A}_{V_{L}}$, i.e. $\Phi\left(X+e_{1}\right)=T^{*} \Phi(X) T$ for all $X \subset V_{L}$. This unitary $T$ will depend on the length scale $L$, but we will suppress this in our notation.

Assumption II. We further assume that the interaction has a finite range $R>0$ in the horizontal direction. This assumption is not strictly necessary. It is clear from the estimates in [23] that the result remains true even if the interactions are of long range with sufficiently fast decay.

Assumption III. We assume the interaction $\Phi$ has rotation invariance about one axis. Again, this is clearly the case for the Heisenberg model. In the more abstract setting, we specifically assume that for each $x \in V$ there is a local hermitian matrix, which we will denote by $S_{x}^{3}$, with eigenvalues that are either all integer or all half-integer. These matrices are also required to be translates of one another, i.e., for any $x \in V, \alpha_{1}\left(S_{x}^{3}\right)=S_{x+e_{1}}^{3}$. Rotation invariance for a general interaction $\Phi$ means that for any $X \subset V_{L}$,

$$
U^{*}(\theta) \Phi(X) U(\theta)=\Phi(X) \text { for all } \theta \in \mathbb{R},
$$

where the rotation $U(\theta)$ is defined by

$$
U(\theta)=\bigotimes_{x \in V_{L}} e^{i \theta S_{x}^{3}}
$$

Assumption IV. We assume that the matrices introduced above, i.e. $S_{x}^{3}$, are uniformly bounded in the sense that there exists a positive real number $S$ for which $\sup _{x \in V}\left\|S_{x}^{3}\right\| \leq S$. In addition, we must assume an odd parity condition on the spins. Define the parity $p_{x}$ of a site $x \in V$ to be 0 if the eigenvalues of $S_{x}^{3}$ are all integers and $1 / 2$ if they are all half-integers. The odd parity assumption is that

$$
\sum_{v \in V_{L}^{\perp}} p_{(n, v)} \in \mathbb{N}+1 / 2
$$

for all $n \in \mathbb{Z}$. For the spin-1/2 Heisenberg model, we satisfied this assumption by taking the cardinality of the perpendicular sets, $\left|V_{L}^{\perp}\right|$, to be odd. In general, the sum of the spins over the perpendicular set needs to be half-integer to ensure that the column rotations $U_{n}(\theta)$, as defined after equation (82), satisfy $U_{n}(2 \pi)=-1$. As we have seen, such an identity plays a crucial role in our argument for orthogonality.

Assumption V. The ground state of $H_{L}$ is assumed to be non-degenerate. In this case, it is also an eigenvector of the translation $T$ and the rotations $U(\theta)$ introduced above. We assume that the ground state has eigenvalue one for both $T$ and $U(\theta)$.

Assumption VI. We assume that there are orthonormal bases of the Hilbert spaces $\mathcal{H}_{V_{L}}$ with respect to which $S_{x}^{3}$ and $\Phi(X)$ are real for all $x \in V$ and all finite $X \subset V$. This assumption is satisfied by the Heisenberg model, and it is an important symmetry which allows us, in general, to prove that the ground state eigenvalue is invariant with respect to the doubly twisted Hamiltonian $H_{\theta,-\theta}$.

The following theorem was proven in 23$]$.

Theorem 5.6 Let $\Phi \in \mathcal{B}_{a}(V)$ for some $a>0$. If $\Phi$ satisfies assumptions I-VI above, then the gap, $\gamma_{L}$, above the ground state energy of $H_{L}$ satisfies

$$
\gamma_{L} \leq C \frac{\log (L)}{L}
$$


Acknowledgments: R.S. would like to acknowledge the gracious hospitality provided by the Isaac Newton Institute for Mathematical Sciences where the writing of this work began. This article is based on work supported by the U.S. National Science Foundation under Grant \# DMS-0605342. A portion of this work was also supported by the Austrian Science Fund (FWF) under Grant No. Y330.

\section{References}

[1] Affleck, I. and Lieb, E.: A proof of part of Haldane's conjecture on quantum spin chains, Lett. Math. Phys. 12, 57-69 (1986).

[2] Araki, H., Hepp, K., Ruelle, D.: Asymptotic behaviour of Wightman functions, Helv. Phys. Acta 35, 164 (1962)

[3] Araki, H.: Gibbs States of a One Dimensional Quantum Lattice, Commun. Math. Phys. 14, 120-157 (1969).

[4] Bratteli, O. and Robinson D.W.: Operator Algebras and Quantum Statistical Mechanics. Volume 2., Second Edition. Springer-Verlag, 1997.

[5] Bravyi, S. and Hastings, M.B. and Verstraete, F.: Lieb-Robinson bounds and the generation of correlations and topological quantum order, Phys. Rev. Lett. 97, 050401 (2006), arXiv:quant-ph/0603121.

[6] Eisert, J. and Osborne, T.J.: General entanglement scaling laws from time evolution, Phys. Rev. Lett. 97, 150404 (2006), arXiv:quant-ph/0603114.

[7] Fredenhagen, K.: A Remark on the Cluster Theorem, Commun. Math. Phys. 97, 461-463 (1985).

[8] Haag, R.: Local Quantum Physics, Springer-Verlag, 1992.

[9] Haldane, F. D. M.: Continuum dynamics of the 1-D Heisenberg antiferromagnet: Identification with the $O(3)$ nonlinear sigma model. Phys. Lett. 93A, 464-468 (1983).

[10] Hastings, M.B.: Lieb-Schultz-Mattis in Higher Dimensions, Phys. Rev. B 69, 104431 (2004).

[11] Hastings, M.B. and Wen, X.-G.: Quasi-adiabatic continuation of quantum states: The stability of topological ground-state degeneracy and emergent gauge invariance, Phys. Rev. B 72, 045141 (2005).

[12] Hastings, M.B. and Koma, T.: Spectral Gap and Exponential Decay of Correlations, Commun. Math. Phys. 265, 781-804 (2006).

[13] Keyl, M., Matsui, T., Schlingemann, D., and Werner, R.: Entanglement, Haag-duality and type properties of infinite quantum spin chains. arXive math-ph/0604071.

[14] Lieb, E.H. and Mattis, D.: Ordering energy levels in interacting spin chains, Journ. Math. Phys. 3, 749-751 (1962).

[15] Lieb, E.H., Schultz, T., and Mattis, D.: Two soluble models of an antiferromagnetic chain, Ann. Phys. 16, 407-466 (1961). 
[16] Lieb, E.H. and Robinson, D.W.: The Finite Group Velocity of Quantum Spin Systems, Commun. Math. Phys. 28, 251-257 (1972).

[17] Liggett, T.M.: Interacting Particle Systems, Springer Verlag 1985.

[18] Nachtergaele, B.: The spectral gap for some quantum spin chains with discrete symmetry breaking, Commun. Math. Phys. 175, 565-606 (1996).

[19] Nachtergaele, B., Ogata, Y., and Sims, R.: Propagation of Correlations in Quantum Lattice Systems, J. Stat. Phys. 124, 1-13 (2006).

[20] Nachtergaele, B., Raz, H., Schlein, B., and Sims, R., Lieb-Robinson Bounds for Harmonic and Anharmonic Lattice Systems, in preparation.

[21] Nachtergaele, B. and Sims, R.: Lieb-Robinson Bounds and the Exponential Clustering Theorem, Commun. Math. Phys. 265, 119-130 (2006).

[22] Nachtergaele, B. and Sims, R.: Recent Progress in Quantum Spin Systems, Markov Processes Relat. Fields 13, 315-329 (2007).

[23] Nachtergaele, B. and Sims, R.: A Multi-Dimesional Lieb-Schultz-Mattis Theorem, Commun. Math. Phys. 276, 437-472 (2007).

[24] Ruelle, D.: On the asymptotic condition in quantum field theory, Helv. Phys. Acta 35, 147 (1962).

[25] Simon, B.: The Statistical Mechanics of Lattice Gases, Volume I, Princeton University Press, 1993.

[26] Wreszinski, W.: Charges and Symmmetries in Quantum Theories without Locality, Fortschr. Phys. 35, 379-413 (1987). 